\title{
Exact solutions of asymmetric baroclinic quasi-geostrophic dipoles with distributed potential vorticity
}

\author{
A. Viúdez ${ }^{1} \dagger$ \\ ${ }^{1}$ Department of Physical Oceanography and Technology, Institute of Marine Sciences, CSIC, \\ Barcelona 08003, Spain
}

(Received $\mathrm{xx}$; revised $\mathrm{xx}$; accepted $\mathrm{xx}$ )

An exact solution of a baroclinic three-dimensional vortex dipole in geophysical flows with constant background rotation and constant background stratification is provided under the quasi-geostrophic $(\mathrm{QG})$ approximation. The motion of the dipole is unsteady but the potential vorticity contours move rigidly. The vortex comprises three potential vorticity anomaly modes, with radial dependence given by the spherical Bessel functions and with azimuthal and polar dependence given by the spherical harmonics. The first mode, or spherical mode, accounts for the horizontal asymmetry of the vortex dipole and curvature of the dipole's horizontal trajectory. The second mode, or dipolar mode, accounts for the speed of displacement of the vortex dipole. A third mode, or vertical tilting mode, accounts for the dipole's vertical asymmetry. The QG vertical velocity field has two contributions, the first one is octupolar and depends entirely on the dipolar mode, and the second one is dipolar and depends on the non-linear interaction between dipolar and vertical tilting modes.

Key words:

\section{Introduction}

Observations of the Earth's oceans and atmosphere at spatial scales larger than the Rossby number reveal the persistence of traveling stable baroclinic vortex pairs of opposite signed vertical component of vorticity. These three-dimensional baroclinic dipoles have been experimentally described from in situ measurements (e.g. de Ruijter et al. 2004), and its surface signature from remote sensing (e.g. Fedorov \& Ginsburg 1989; Ahlnäs et al. 1987; Cunningham \& Keyser 2000) measurements, as coherent, approximately balanced (void of inertia-gravity waves) flow structures where the quasigeostrophic (QG) theory (e.g. Cavallini \& Crisciani 2013) is approximately valid. Laboratory experiments and numerical simulations have confirmed the stability of these dipoles and described their behaviour and interaction with other vortices (e.g. Voropayev \& Afanasyev 1992; Velasco Fuentes \& van Heijst 1995) and with coastal or bottom topography (Kloosterziel et al. 1993). However, despite its physical relevance and the existence of two-layer solutions (Flierl et al. 1980), a mathematical explanation of these baroclinic, continuously stratified, isolated dipoles, is still lacking. This work provides exact mathematical solutions, under the QG regime, of these unsteady vortex structures.

$\dagger$ Email address for correspondence: aviudez@cmima.csic.es 
The procedure followed here to obtain the three-dimensional (3D) unsteady dipole solutions, in terms of a piecewise geopotential anomaly $\phi(\boldsymbol{x}, t)$, is similar to the approach followed in two-dimensional (2D) flows in terms of a piecewise stream function $\phi_{h}\left(\boldsymbol{x}_{h}, t\right)$ leading to the 2D Chaplygin-Lamb dipole (Chaplygin 1903; Meleshko \& van Heijst 1994; Flierl et al. 1983; Viúdez 2019). In the 2D case the dynamics is governed by the material conservation of vorticity $\zeta=\nabla_{h}^{2} \phi_{h}$ by the horizontal flow $\boldsymbol{u}_{h}=\boldsymbol{e}_{z} \times \boldsymbol{\nabla}_{h} \phi_{h}$, while in the QG 3D case the dynamics is governed by the conservation of QG potential vorticity anomaly $(\mathrm{PVA}) \varpi^{q}=\hat{\nabla}^{2} \phi$ by the horizontal geostrophic flow $\boldsymbol{u}_{h}^{g}=\boldsymbol{e}_{z} \times \boldsymbol{\nabla}_{h} \phi$. Above $\hat{\boldsymbol{\nabla}}$ is the gradient operator in the vertically stretched QG space $(x, y, \hat{z})$ where the vertical coordinate $\hat{z} \equiv c z$, and $c \equiv N / f=\epsilon^{-1}$ is the ratio between the constant background Brunt-Väisälä $N$ and Coriolis $f$ frequencies. In the QG 3D case both geostrophic vertical vorticity and vertical density stratification are given by the QG geopotential $\phi$.

The basic QG equations are introduced in $\S 2$. The steady solutions of a baroclinic vortex are found $(\S 3)$ in terms of a piecewise geopotential $\phi(\boldsymbol{x})$ composed of an interior vortex solution $\phi_{i}(\boldsymbol{x})$ with distributed PVA, and an exterior vortex solution $\phi_{e}(\boldsymbol{x})$ with constant (including vanishing) PVA. In order to satisfy the material conservation of the QG PVA the interior geopotential $\phi_{i}$ must be a solution to the Helmholtz equation $\hat{\nabla}^{2} \phi=-k^{2} \phi$, where $k$ is a constant. Assuming separation of variables in spherical coordinates $(\rho, \theta, \varphi)$ the interior solution is a sum of modes with the radial part given by the spherical Bessel functions of the first kind $\mathrm{j}_{l}(\rho)$ and the angular part given by the spherical harmonics $\mathrm{Y}_{l}^{m}(\theta, \varphi)$ of degree $l$ and order $m$. The $\mathrm{QG}$ baroclinic dipole is defined using three modes (for ease of reference named modes-0, 1 and 2 ). The mode- 0 , or spherical mode, has degree $l=0$ (and order $m=0$ ), The mode-1, or dipolar mode, corresponds to the degree $l=1$ and order $m=1$ (or equivalently order $m=-1$ ). The mode-2, or vertical tilting mode, corresponds to the degree $l=1$ and order $m=0$. The QG vertical velocity field $w^{q}$ is also explicitly found. Finally, concluding remarks are given in $\S 4$.

\section{Basic QG dynamics}

The inviscid adiabatic QG flow is governed by the conservation of QG PVA $\varpi^{q}(\boldsymbol{x}, t)$ by the horizontal geostrophic flow (scaled by $\left.f^{-1}\right) \boldsymbol{u}^{g}(\boldsymbol{x}, t) \equiv-\boldsymbol{\nabla}_{h} \times\left(\phi \boldsymbol{e}_{z}\right)$,

$$
\frac{\mathrm{d}_{g} \varpi^{q}}{\mathrm{~d} t} \equiv \frac{\partial \varpi^{q}}{\partial t}+\boldsymbol{u}^{g} \cdot \nabla_{h} \varpi^{q}=0 .
$$

The QG PVA $\varpi^{q}(\boldsymbol{x}, t)$ is the sum of the dimensionless (scaled by $f^{-1}$ ) vertical component of geostrophic vorticity $\zeta^{g}(\boldsymbol{x}, t)=\nabla_{h}^{2} \phi$ and the dimensionless vertical stratification anomaly $\mathcal{S}(\boldsymbol{x}, t)=-\partial \mathcal{D}(\boldsymbol{x}, t) / \partial z=\partial^{2} \phi / \partial \hat{z}^{2}$, where $\mathcal{D}$ is the vertical displacement of isopycnals (Appendix A). The QG PVA $\varpi^{q}$ equals, in the vertically stretched QG space $(x, y, \hat{z})$, the laplacian of the geopotential anomaly $\phi(\boldsymbol{x}, t)$

$$
\varpi^{q}=\zeta^{g}+\mathcal{S}=\hat{\nabla}^{2} \phi .
$$

In terms of the geopotential $\phi(\boldsymbol{x}, t)$ the QG PVA conservation $(2.1)$ is

$$
\frac{\partial \hat{\nabla}^{2} \phi}{\partial t}+\left(\boldsymbol{e}_{z} \times \nabla_{h} \phi\right) \cdot \nabla_{h} \hat{\nabla}^{2} \phi=0 .
$$

Steady state solutions to (2.3) imply vectors $\boldsymbol{e}_{z} \times \nabla_{h} \phi$ and $\nabla_{h} \hat{\nabla}^{2} \phi$ be perpendicular, which is satisfied for rotational flows if $\hat{\nabla}^{2} \phi=F(\phi)$, with $F$ an arbitrary function. We, however, do not impose solutions satisfying $\hat{\nabla}^{2} \phi=F(\phi)$, but rather derive it as a 
property, for linear $F(\phi)$, assuming time-space variables separation and azimuthal-mode spatial dependence.

\section{The asymmetric QG dipole solution}

\subsection{Steady solutions}

In this section we employ spherical coordinates in the $\mathrm{QG}$ space $(x, y, \hat{z})$ with radial distance $\rho(x, y, \hat{z}) \equiv \sqrt{x^{2}+y^{2}+\hat{z}^{2}}$, colatitude (polar angle) $\theta \equiv \arccos (\hat{z} / \rho(x, y, \hat{z}))$, and longitude (azimuthal angle) $\varphi \equiv \arctan (y / x)$. Assuming separation of space and time variables in the geopotential $\Phi(\rho, \theta, \varphi, t)=\phi(\rho, \theta, \varphi) T(t)$, conservation of PVA $(2.3)$ leads to steady state $(T(t)$ is constant) geopotential solutions $\phi(\rho, \theta, \varphi)$. If, furthermore, azimuthal modes dependence is assumed $\phi(\rho, \theta, \varphi)=G(\rho, \theta) \mathrm{e}^{\mathrm{i} m \varphi}$ then $\phi(\rho, \theta, \varphi)$ (Appendix B) satisfies Helmholtz equation

$$
\hat{\nabla}^{2} \phi=-k^{2} \phi
$$

The interior geopotential $\phi_{i}(\rho, \theta, \varphi)$ is assumed to have distributed PVA $\varpi^{q}$ so that it satisfies (3.1) whose solution is an infinite sum of modes

$$
\phi_{i}(\rho, \theta, \varphi)=\sum_{l=0}^{\infty} \sum_{m=-l}^{l} \phi_{l m}(\rho, \theta, \varphi)=\sum_{l=0}^{\infty} \sum_{m=-l}^{l}\left(a_{l m} \mathrm{j}_{l}(k \rho)+b_{l m} \mathrm{y}_{l}(k \rho)\right) \mathrm{Y}_{l}^{m}(\theta, \varphi),
$$

where $\mathrm{j}_{l}(k \rho)$ and $\mathrm{y}_{l}(k \rho)$ are the spherical Bessel functions of the first and second kind, respectively, and $\mathrm{Y}_{l}^{m}(\theta, \varphi)$ are the spherical harmonics of degree $l$ and order $m$, $\mathrm{Y}_{l}^{m}(\theta, \varphi)=N_{l m} \mathrm{e}^{\mathrm{i} m \varphi} \mathrm{P}_{l}^{m}(\cos \theta)$ where $\mathrm{P}_{l}^{m}(\cos \theta)$ is the associated Legendre polynomial, and $N_{l m}$ is a normalization constant. The domain of the interior solution includes the origin point $\rho=0$ and consequently functions $\mathrm{y}_{l}(k \rho)$, which are singular at the origin, are ruled out from the interior geopotential solution (setting $b_{l m}=0$ ).

From the complete set of modes in (3.2) we consider here only three modes. The simplest QG dipole solution (referred to as mode-1 and labeled with subscript 1) is the mode with degree $l=1$ and order $m=1$, whose interior geopotential has an azimuthal and polar dependence given by $\cos \varphi \sin \theta$. Mode with order $m=-1$ is only a $\pi / 2$ azimuthal rotation of order $m=1$ and will be obviated. We choose therefore the dipole's orientation such that the plane of symmetry of mode- 1 is the plane $x=0$ (the dipole's horizontal axis is the $y$-axis). Mode $l=0$ (referred to as mode- 0 and labeled with subscript 0 ) has only radial dependence and is included in order to take into account dipoles with horizontal asymmetry. The third mode (mode-2, labeled with subscript 2 ) is the mode with degree $l=1$ and order $m=0$ whose angular dependence $\cos \theta$ is only polar. The geostrophic flow of mode-2, like that of mode-0, has circular stream lines. This third mode is included in order to take into account a possible dipole's vertical asymmetry.

For simplicity, the product of the relevant coefficients $a_{l m}$ in (3.2) with the constants $N_{l m}$ of $\mathrm{Y}_{l}^{m}(\theta, \varphi)$ are merged into one single constant which denotes the amplitude of their respective modes, that is $\left\{\hat{\phi}_{0}, \hat{\phi}_{1}, \hat{\phi}_{2}\right\}=\left\{a_{0,0} N_{0,0}, a_{1,1} N_{1,1}, a_{1,0} N_{1,0}\right\}$.

The radius separating the interior (henceforth subscript $i$ ) from the exterior (henceforth subscript $e$ ) dipole solutions is set to the first zero $\rho_{1}$ of the spherical Bessel function of the dipole mode $\mathrm{j}_{1}(\rho)$, thus $\mathrm{j}_{1}\left(\rho_{1}\right)=0$, and for convenience we choose the spatial scale parameter $k=1$ so that the radius $\rho_{1} \simeq 4.49341$ sets the dipole vortex extent. The spherical Bessel functions of degrees $l=0, l=1, l=2$, and $l=3$ appearing in the following mathematical development are explicitly given in Appendix C.

The exterior solutions $\phi_{e}$ are assumed to have homogeneous QG PVA $\varpi^{q}$. The polar and azimuthal terms of $\phi_{e}$ are the corresponding spherical harmonics $\mathrm{Y}_{l}^{m}(\theta, \varphi)$ of the 
(a)

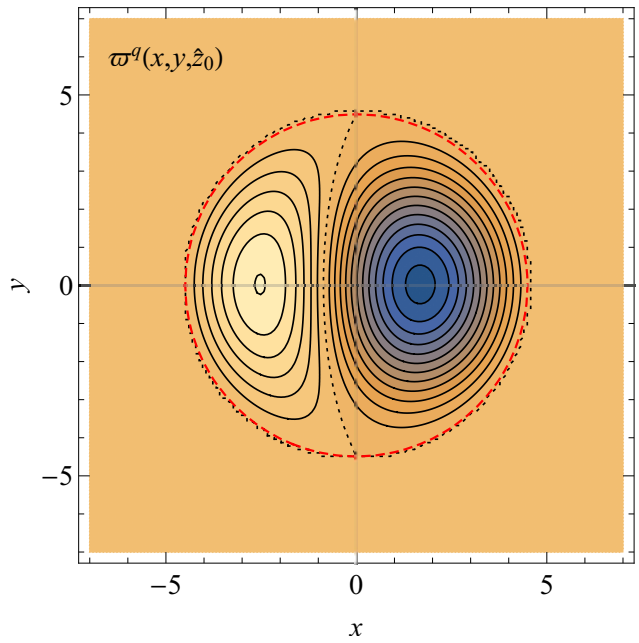

(b)

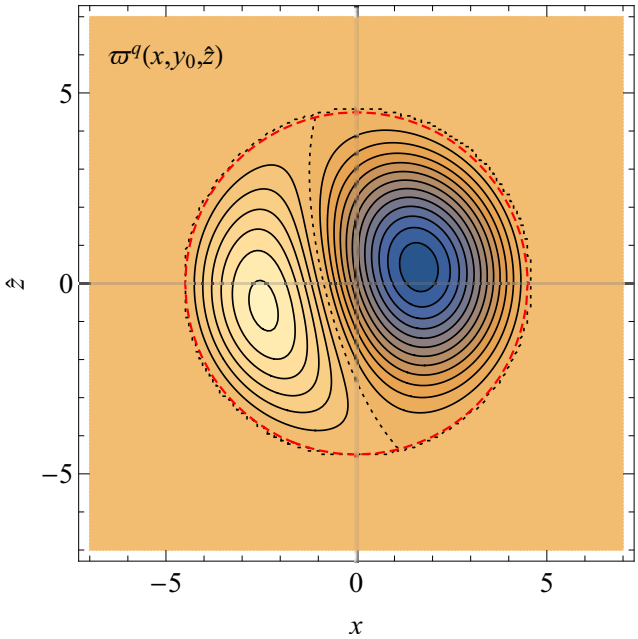

Figure 1. (a) Horizontal distribution on plane $\hat{z}_{0}=0$, and $(b)$ vertical distribution on plane $y_{0}=0$ of $\varpi(x, y, \hat{z})+\hat{\phi}_{0} \mathrm{j}_{0}\left(\rho_{1}\right)$, which equals the unsteady QG PVA $\widetilde{\varpi}\left(x, y, \hat{z}, t_{0}\right)$ at time $t_{0}=0$. Amplitudes $\left\{\hat{\phi}_{0}, \hat{\phi}_{1}, \hat{\phi}_{2}\right\}=\{0.05,0.2,0.05\}$. Contour interval $\Delta=0.01$. In all figures the red dashed circle, with radius $\rho=\rho_{1} \simeq 4.49$, separates the interior from the exterior solution. Blue colors in all figures correspond to negative values with the dotted contour meaning zero values.

interior solutions $\phi_{i}$, while the radial dependence is given by the sum of an harmonic contribution $a_{l} \rho^{l}+b_{l} \rho^{-l-1}$ satisfying $\left(\rho^{2} R_{l}^{\prime}(\rho)\right)^{\prime}=l(l+1) R_{l}$, and a term $c_{l} \rho^{2}$ given a constant PVA,

$$
R_{e l}(\rho)=a_{l} \rho^{l}+b_{l} \rho^{-l-1}+c_{l} \rho^{2} .
$$

The constant coefficients $\left\{a_{l}, b_{l}, c_{l}\right\}$ are chosen to ensure that $\phi_{l i}=\phi_{l e}, \partial \phi_{l i} / \partial \rho=$ $\partial \phi_{l e} / \partial \rho$, and $\partial^{2} \phi_{l i} / \partial \rho^{2}=\partial^{2} \phi_{l e} / \partial \rho^{2}$ evaluated at the boundary $\rho=\rho_{1}$ for degrees $l=0$ and $l=1$. These matching conditions ensure that the geostrophic velocity $\boldsymbol{u}^{g}=\boldsymbol{e}_{z} \times \boldsymbol{\nabla}_{h} \phi$, geostrophic vertical vorticity $\zeta^{g}=\nabla_{h}^{2} \phi$, and vertical stratification anomaly $\mathcal{S}=\partial^{2} \phi / \partial \hat{z}^{2}$ are continuous at the vortex boundary radius $\rho=\rho_{1}$ for the three modes.

Finally, the piecewise QG dipole geopotential is the sum of the three modes

$$
\phi(\rho, \theta, \varphi)=\phi_{0}(\rho)+\phi_{1}(\rho, \theta, \varphi)+\phi_{2}(\rho, \theta)
$$

where the piecewise geopotential modal components are

$$
\begin{aligned}
& \frac{\phi_{0}(\rho)}{\hat{\phi}_{0}}= \begin{cases}\mathrm{j}_{0}(\rho), & \rho \leqslant \rho_{1} \\
\mathrm{j}_{0}\left(\rho_{1}\right)\left(1-\frac{\left(\rho_{1}-\rho\right)^{2}\left(2 \rho_{1}+\rho\right)}{6 \rho}\right), & \rho_{1}<\rho,\end{cases} \\
& \frac{\phi_{1}(\rho, \theta, \varphi)}{\hat{\phi}_{1} \sin \theta \cos \varphi}=\frac{\phi_{2}(\rho, \theta)}{\hat{\phi}_{2} \cos \theta}= \begin{cases}\mathrm{j}_{1}(\rho), & \rho \leqslant \rho_{1} \\
\frac{\mathrm{j}_{0}\left(\rho_{1}\right)}{3} \frac{\rho^{3}-\rho_{1}^{3}}{\rho^{2}}, & \rho_{1}<\rho .\end{cases}
\end{aligned}
$$

Mode- 0 is only radial, while mode- 2 has not azimuthal dependence. Geopotentials are continuous at the vortex boundary $\rho=\rho_{1}$ with $\phi_{0} i\left(j_{1}\right)=\phi_{0} e\left(j_{1}\right)=\mathrm{j}_{0}\left(\rho_{1}\right)$, and $\phi_{1 i}\left(j_{1}\right)=$ $\phi_{1 e}\left(j_{1}\right)=\phi_{2 i}\left(j_{1}\right)=\phi_{2 e}\left(j_{1}\right)=0$.

The QG PVA $\varpi^{q}$, from (2.2) and (3.5)-(3.6), is

$$
\varpi^{q}(\rho, \theta, \varphi)= \begin{cases}-\hat{\phi}_{0} \mathrm{j}_{0}(\rho)-\hat{\phi}_{1} \mathrm{j}_{1}(\rho) \sin \theta \cos \varphi-\hat{\phi}_{2} \mathrm{j}_{1}(\rho) \cos \theta, & \rho \leqslant \rho_{1} \\ -\hat{\phi}_{0} \mathrm{j}_{0}\left(\rho_{1}\right), & \rho_{1}<\rho .\end{cases}
$$


(a)

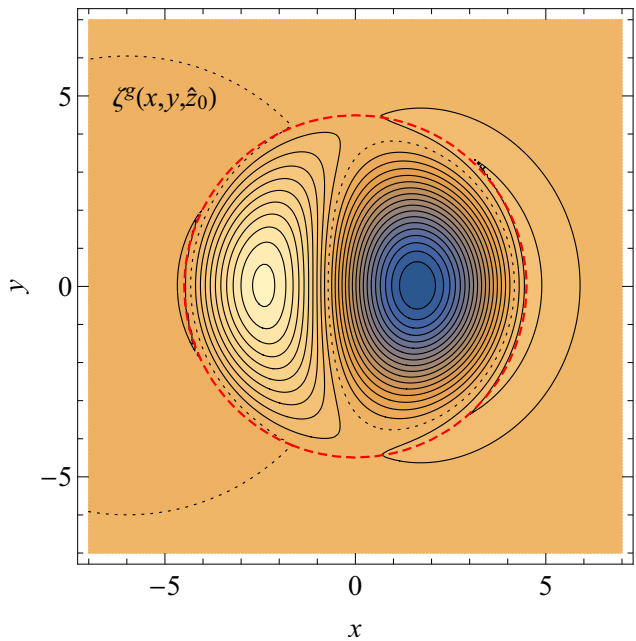

(b)

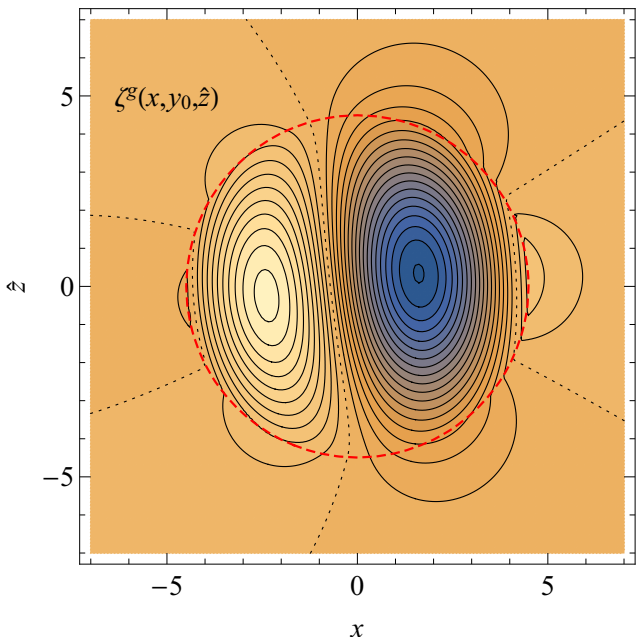

Figure 2. (a) Horizontal distribution on plane $\hat{z}_{0}=0$, and $(b)$ vertical distribution on plane $y_{0}=0$ of $\zeta^{g}(x, y, \hat{z})+(2 / 3) \hat{\phi}_{0} \mathrm{j}_{0}\left(\rho_{1}\right)$, which equals the unsteady vorticity $\widetilde{\zeta}^{g}\left(x, y, \hat{z}, t_{0}\right)$ at time $t_{0}=0$. Amplitudes $\left\{\hat{\phi}_{0}, \hat{\phi}_{1}, \hat{\phi}_{2}\right\}=\{0.05,0.2,0.05\}$. Contour interval $\Delta=0.005$.

Clearly, due to (3.1), the interior PVA $\varpi_{i}^{q}(\rho, \theta, \varphi)=-\phi_{i}(\rho, \theta, \varphi)$, while the exterior PVA $\varpi_{e}^{q}=\varpi_{i}^{q}\left(\rho_{1}, \theta, \varphi\right)=-\hat{\phi}_{0} \mathrm{j}_{0}\left(\rho_{1}\right)$ is a constant depending only on mode-0 since modes 1 and 2 have vanishing exterior PVA due to the boundary conditions $\varpi_{1 i}=\varpi_{1 e}=\varpi_{2 i}=$ $\varpi_{2 e}=0$ at $\rho=\rho_{1}$ (figure 1 ). To lighten the notation the domain specifications are henceforth omitted in the piecewise function expressions and it is implicitly understood that the domains of the upper and lower functions are $\rho \leqslant \rho_{1}$ and $\rho_{1}<\rho$, respectively.

The geostrophic velocity $\boldsymbol{u}^{g} \equiv \boldsymbol{e}_{z} \times \boldsymbol{\nabla}_{h} \phi$ of the three modes is

$$
\begin{aligned}
& \frac{\boldsymbol{u}_{0}^{g}(\rho, \theta)}{\hat{\phi}_{0}}=\sin \theta \boldsymbol{e}_{\varphi}\left\{\begin{array}{l}
-\mathrm{j}_{1}(\rho) \\
\frac{\mathrm{j}_{0}\left(\rho_{1}\right)}{3} \frac{\rho_{1}^{3}-\rho^{3}}{\rho^{2}},
\end{array}\right. \\
& \frac{\boldsymbol{u}_{1}^{g}(\rho, \theta, \varphi)}{\hat{\phi}_{1}}=\left\{\begin{array}{l}
\frac{\mathrm{j}_{1}(\rho)}{\rho} \sin \varphi \boldsymbol{e}_{r}+\left(\frac{\mathrm{j}_{1}(\rho)}{\rho}-\mathrm{j}_{2}(\rho) \sin ^{2} \theta\right) \cos \varphi \boldsymbol{e}_{\varphi} \\
\frac{\mathrm{j}_{0}\left(\rho_{1}\right)}{3}\left[\frac{\rho^{3}-\rho_{1}^{3}}{\rho^{3}} \sin \varphi \boldsymbol{e}_{r}+\left(\frac{\rho_{1}^{3}}{\rho^{3}}\left(3 \sin ^{2} \theta-1\right)+1\right) \cos \varphi \boldsymbol{e}_{\varphi}\right],
\end{array}\right. \\
& \frac{\boldsymbol{u}_{2}^{g}(\rho, \theta)}{\hat{\phi}_{2}}=\sin \theta \cos \theta \boldsymbol{e}_{\varphi}\left\{\begin{array}{l}
-\mathrm{j}_{2}(\rho) \\
\mathrm{j}_{0}\left(\rho_{1}\right) \frac{\rho_{1}^{3}}{\rho^{3}},
\end{array}\right.
\end{aligned}
$$

where $\boldsymbol{e}_{r}=\sin \theta \boldsymbol{e}_{\rho}+\cos \theta \boldsymbol{e}_{\theta}$ is the horizontal radial unit vector, so that (3.8)-(3.10) express the cylindrical vector components in spherical coordinates. Mode- 0 and 2 have circular streamlines but in mode-2, due to the $\cos \theta$ dependence, the directions of the flow are opposite in the upper $(z>0)$ and lower $(z<0)$ domains.

At the vortex boundary $\rho=\rho_{1}$ the total geostrophic velocity $\boldsymbol{u}^{g}$ is azimuthal: the mode-0 velocity vanishes $\boldsymbol{u}_{0 i}^{g}=\boldsymbol{u}_{0 e}^{g}=\mathbf{0}$, the mode-1 velocity $\boldsymbol{u}_{1 i}^{g}=\boldsymbol{u}_{1 e}^{g}=$ $\hat{\phi}_{1} \mathrm{j}_{0}\left(\rho_{1}\right) \sin ^{2} \theta \cos \varphi \boldsymbol{e}_{\varphi}$, has polar and azimuthal dependence, and the mode-2 velocity $\boldsymbol{u}_{2 i}^{g}=\boldsymbol{u}_{2 e}^{g}=\hat{\phi}_{2} \mathrm{j}_{0}\left(\rho_{1}\right) \sin \theta \cos \theta \boldsymbol{e}_{\varphi}$ has only polar dependence. At the dipole's centre $(\rho=0)$ velocity $\boldsymbol{u}^{g}$ is only due to mode- 1 . Since $\lim _{\rho \rightarrow 0} \mathbf{j}_{1}(\rho) / \rho=1 / 3$ the velocity at the dipole's origin is $\boldsymbol{u}^{g}(0)=\left(\hat{\phi}_{1} / 3\right)\left(\sin \varphi \boldsymbol{e}_{r}+\cos \varphi \boldsymbol{e}_{\varphi}\right)=\left(\hat{\phi}_{1} / 3\right) \boldsymbol{e}_{y}$. 
The modal components of the vertical displacement of isopycnals $\mathcal{D} \equiv-\epsilon \partial \phi / \partial \hat{z}$ are

$$
\begin{aligned}
& \frac{\mathcal{D}_{0}(\rho, \theta)}{\epsilon \hat{\phi}_{0}}=\cos \theta\left\{\begin{array}{l}
\mathrm{j}_{1}(\rho) \\
\frac{\mathrm{j}_{0}\left(\rho_{1}\right)}{3} \frac{\rho^{3}-\rho_{1}^{3}}{\rho^{2}},
\end{array} \quad \frac{\mathcal{D}_{1}(\rho, \theta, \varphi)}{\epsilon \hat{\phi}_{1}}=\sin \theta \cos \theta \cos \varphi\left\{\begin{array}{l}
\mathrm{j}_{2}(\rho) \\
-\mathrm{j}_{0}\left(\rho_{1}\right) \frac{\rho_{1}^{3}}{\rho^{3}},
\end{array}\right.\right. \\
& \frac{\mathcal{D}_{2}(\rho, \theta)}{\epsilon \hat{\phi}_{2}}=\left\{\begin{array}{l}
-\frac{\mathrm{j}_{1}(\rho)}{\rho}+\mathrm{j}_{2}(\rho) \cos ^{2} \theta, \\
-\mathrm{j}_{0}\left(\rho_{1}\right)\left(\frac{\rho^{3}-\rho_{1}^{3}}{3 \rho^{3}}+\frac{\rho_{1}^{3}}{\rho^{3}} \cos ^{2} \theta\right),
\end{array}\right.
\end{aligned}
$$

the geostrophic vertical vorticity $\zeta^{g}=\nabla_{h}^{2} \phi$ (figure 2 ) is

$$
\begin{aligned}
& \frac{\zeta_{0}^{g}(\rho, \theta)}{\hat{\phi}_{0}}=\left\{\begin{array}{l}
-2 \frac{\mathrm{j}_{1}(\rho)}{\rho}+\mathrm{j}_{2}(\rho) \sin ^{2} \theta \\
\frac{\mathrm{j}_{0}\left(\rho_{1}\right)}{3}\left(\frac{\rho_{1}^{3}}{\rho^{3}}\left(2-3 \sin ^{2} \theta\right)-2\right),
\end{array}\right. \\
& \frac{\zeta_{2}^{g}(\rho, \theta)}{\hat{\phi}_{2} \cos \theta}=\left\{\begin{array}{l}
-2 \frac{\zeta_{1}^{g}(\rho, \theta, \varphi)}{\hat{\phi}_{1} \sin \theta \cos \varphi}+\mathrm{j}_{3}(\rho) \sin ^{2} \theta \\
\rho_{1}^{3} \mathrm{j}_{0}\left(\rho_{1}\right) \frac{2-5 \sin ^{2} \theta}{\rho^{4}},
\end{array}\right.
\end{aligned}
$$

and the dimensionless vertical stratification $\mathcal{S}=\partial^{2} \phi / \partial \hat{z}^{2}$,

$$
\begin{aligned}
\frac{\mathcal{S}_{0}(\rho, \theta)}{\hat{\phi}_{0}}=\left\{\begin{array}{l}
-\mathrm{j}_{0}(\rho)+2 \frac{\mathrm{j}_{1}(\rho)}{\rho}-\mathrm{j}_{2}(\rho) \sin ^{2} \theta \\
-\frac{\mathrm{j}_{0}\left(\rho_{1}\right)}{3}\left(1+\frac{\rho_{1}^{3}}{\rho^{3}}\left(2-3 \sin ^{2} \theta\right)\right),
\end{array}\right. \\
\frac{\mathcal{S}_{1}(\rho, \theta, \varphi)}{\hat{\phi}_{1} \sin \theta \cos \varphi}=\left\{\begin{array}{l}
-\mathrm{j}_{1}(\rho)+4 \frac{\mathrm{j}_{2}(\rho)}{\rho}-\mathrm{j}_{3}(\rho) \sin ^{2} \theta \\
-\rho_{1}^{3} \mathrm{j}_{0}\left(\rho_{1}\right) \frac{4-5 \sin ^{2} \theta}{\rho^{4}},
\end{array}\right. \\
\frac{\mathcal{S}_{2}(\rho, \theta)}{\hat{\phi}_{2} \cos \theta}=\left\{\begin{array}{l}
-\mathrm{j}_{1}(\rho)+2 \frac{\mathrm{j}_{2}(\rho)}{\rho}-\mathrm{j}_{3}(\rho) \sin ^{2} \theta \\
-\rho_{1}^{3} \mathrm{j}_{0}\left(\rho_{1}\right) \frac{2-5 \sin ^{2} \theta}{\rho^{4}}
\end{array}\right.
\end{aligned}
$$

All the fields above are continuous at $\rho=\rho_{1}$. It is confirmed, from (3.12) and (3.13), that that the sum $\zeta^{g}(\rho, \theta, \varphi)+\mathcal{S}(\rho, \theta, \varphi)$ equals the QG PVA $\varpi^{q}(\rho, \theta, \varphi)(3.7)$. For these flows remain physically acceptable within the QG approximation they should be far from being inertially unstable $\left(\zeta^{g}<-1\right)$ and statically unstable $(\mathcal{S}<-1$, Appendix A). This requirement implies, in general, modal amplitudes $\left|\hat{\phi}_{n}\right| \ll 1$. For example, in mode- 0 the onset of inertial instability $\left(\zeta^{g}=-1\right)$ and static instability $(\mathcal{S}=-1)$ occurs at $\rho=0$ when the amplitude $\hat{\phi}_{0}= \pm 3 / 2$ (notice that $\lim _{\rho \rightarrow 0} \mathrm{j}_{1}(\rho) / \rho=1 / 3$ ).

Fields $\mathcal{D}, \zeta^{g}$, and $\mathcal{S}$ are linear functions of $\phi$ and therefore are the sum of their corresponding modal components. The QG vertical velocity $w^{q}$, which is a diagnostic field in QG theory, is however a nonlinear quantity. In the steady state $w^{q}$ is given (see (A 2)) by the vertical displacement of fluid particles on isopycnals as advected by $\boldsymbol{u}^{g}$

$$
w^{q}=\boldsymbol{u}^{g} \cdot \nabla_{h} \mathcal{D}=\boldsymbol{u}_{1}^{g} \cdot \nabla_{h} \mathcal{D}_{1}+\boldsymbol{u}_{1}^{g} \cdot \nabla_{h} \mathcal{D}_{2}+\boldsymbol{u}_{2}^{g} \cdot \nabla_{h} \mathcal{D}_{1}
$$

Though the three modes are included above in $\boldsymbol{u}^{g}$ and $\mathcal{D}$, which would give rise to nine terms of the form $\boldsymbol{u}_{m}^{g} \cdot \boldsymbol{\nabla}_{h} \mathcal{D}_{n}$, only the three terms on the right-hand side of (3.14) are needed. This is so because mode- 0 and mode- 2 have circular geostrophic streamlines, being $\boldsymbol{u}_{0,2}^{g}$ azimuthal and $\nabla_{h} \mathcal{D}_{0,2}$ radial vector fields. Therefore the following advective terms vanish $\boldsymbol{u}_{0}^{g} \cdot \boldsymbol{\nabla}_{h} \mathcal{D}_{0}=\boldsymbol{u}_{2}^{g} \cdot \nabla_{h} \mathcal{D}_{2}=\boldsymbol{u}_{0}^{g} \cdot \nabla_{h} \mathcal{D}_{2}=\boldsymbol{u}_{2}^{g} \cdot \nabla_{h} \mathcal{D}_{0}=0$. Also, the interaction between mode- 0 and mode- 1 vanishes since $\boldsymbol{u}_{0}^{g} \cdot \nabla_{h} \mathcal{D}_{1}=-\boldsymbol{u}_{1}^{g} \cdot \nabla_{h} \mathcal{D}_{0}$. Thus, $w^{q}$ is the sum of the $\mathrm{QG}$ vertical velocity of mode-1 and the $\mathrm{QG}$ vertical velocity due to its interaction 
(a)

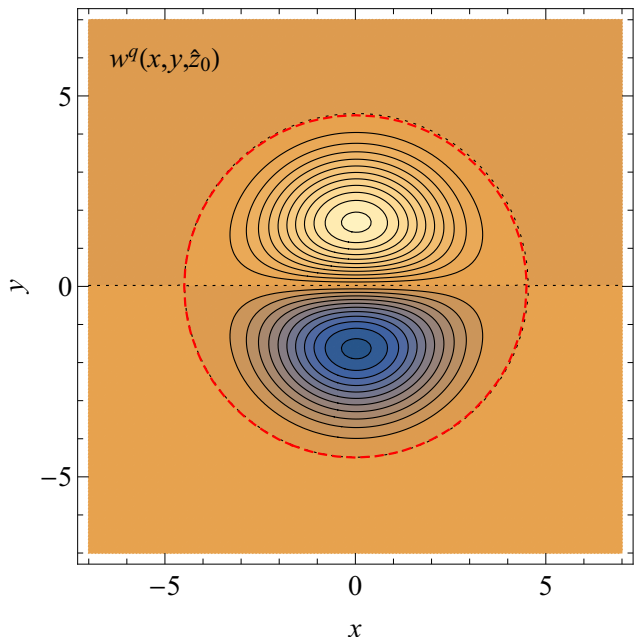

(b)

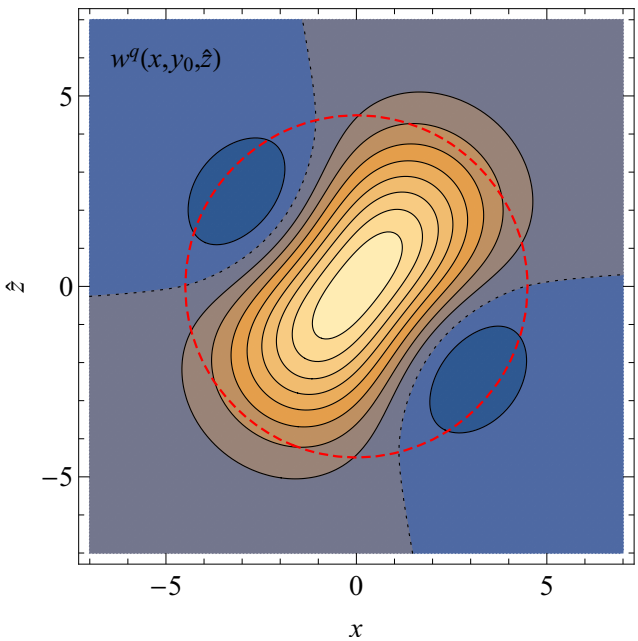

Figure 3. (a) Horizontal distribution on plane $\hat{z}_{0}=0$ (contour interval $\Delta=2 \times 10^{-7}$ ), and (b) vertical distribution on plane $y_{0}=0.2\left(\Delta=5 \times 10^{-8}\right)$ of $w^{q}(x, y, \hat{z})$, which equals the unsteady QG vertical velocity $\widetilde{w}^{q}\left(x, y, \hat{z}, t_{0}\right)$ at time $t_{0}=0$. Amplitudes $\left\{\hat{\phi}_{0}, \hat{\phi}_{1}, \hat{\phi}_{2}\right\}=\{0.05,0.2,0.05\}$.

with mode-2. Explicitly, in spherical coordinates, from (3.9)-(3.10) and (3.11), $w^{q}$ is

$$
\frac{w^{q}(\rho, \theta, \varphi)}{\epsilon \hat{\phi}_{1} \sin \theta \sin \varphi}=\left\{\begin{array}{l}
\hat{\phi}_{1} \frac{\sin (2 \theta)}{2} \cos \varphi R_{i}(\rho)+\hat{\phi}_{2}\left(\frac{\mathrm{j}_{1}(\rho) \mathrm{j}_{2}(\rho)}{\rho^{2}}+R_{i}(\rho) \cos ^{2} \theta\right) \\
\frac{\mathrm{j}_{0}^{2}\left(\rho_{1}\right)}{3} \frac{\rho_{1}^{3}}{\rho^{7}}\left(\hat{\phi}_{1} \frac{\sin (2 \theta)}{2} \cos \varphi R_{e}(\rho)+\hat{\phi}_{2}\left(\rho_{1}^{3}-\rho^{3}+R_{e}(\rho) \cos ^{2} \theta\right)\right),
\end{array}\right.
$$

where the interior and exterior radial functions are

$$
R_{i}(\rho) \equiv \frac{\mathrm{j}_{1}^{2}(\rho)+\mathrm{j}_{2}^{2}(\rho)}{\rho}-5 \frac{\mathrm{j}_{1}(\rho) \mathrm{j}_{2}(\rho)}{\rho^{2}}, \quad R_{e}(\rho) \equiv 5 \rho^{3}-2 \rho_{1}^{3} .
$$

The vertical velocity $w^{q}$ (figure 3 ) is continuous at $\rho=\rho_{1}$ since the terms on the righthand side of the curly bracket in (3.15) are equal to $\left(\mathrm{j}_{0}^{2}\left(\rho_{1}\right) / \rho_{1}\right) \cos \theta\left(\hat{\phi}_{1} \sin \theta \cos \varphi+\right.$ $\left.\hat{\phi}_{2} \cos \theta\right)$. The $w^{q}$ distribution of mode- 1 is octupolar (due to its dependence on $\sin (2 \theta) \sin (2 \varphi))$ and vanishes at the planes $x=y=\hat{z}=0$. However, the $w^{q}$ associated to the interaction between dipolar and tilting modes has a dipolar distribution (due to its dependence on $\sin \varphi$ ) and on plane $\hat{z}=0$ is equal to $w^{q}(\rho, \pi / 2, \varphi)=\epsilon \hat{\phi}_{1} \hat{\phi}_{2} \sin \varphi \mathbf{j}_{1}(\rho) \mathbf{j}_{2}(\rho) / \rho^{2}$.

\subsection{Unsteady solutions}

The steady solutions are not completely satisfactory in the sense that these are not isolated fields in which velocity and density anomaly vanish as $\rho \rightarrow \infty$. From the modal velocities (3.8)-(3.10) and vertical displacement of isopycnals (3.11) the far-field limits or asymptotic behaviour as $\rho \rightarrow \infty$ of the modal velocity and modal vertical displacement of isopycnals are

$$
\begin{aligned}
\boldsymbol{u}_{0}^{g}(\rho, \theta) & \sim-\hat{\phi}_{0} \frac{\mathrm{j}_{0}\left(\rho_{1}\right)}{3} \rho \sin \theta \boldsymbol{e}_{\varphi}=-\hat{\phi}_{0} \frac{\mathrm{j}_{0}\left(\rho_{1}\right)}{3} r(\rho, \theta) \boldsymbol{e}_{\varphi} \equiv \boldsymbol{U}_{0}(\rho, \theta), \\
\lim _{\rho \rightarrow \infty} \boldsymbol{u}_{1}^{g}(\rho, \theta, \varphi) & =\hat{\phi}_{1} \frac{\mathrm{j}_{0}\left(\rho_{1}\right)}{3}\left(\sin \varphi \boldsymbol{e}_{r}+\cos \varphi \boldsymbol{e}_{\varphi}\right)=\hat{\phi}_{1} \frac{\mathrm{j}_{0}\left(\rho_{1}\right)}{3} \boldsymbol{e}_{y} \equiv \boldsymbol{U}_{1}, \\
\lim _{\rho \rightarrow \infty} \boldsymbol{u}_{2}^{g}(\rho, \theta) & =\mathbf{0}
\end{aligned}
$$




$$
\begin{aligned}
\mathcal{D}_{0}(\rho, \theta) & \sim \epsilon \hat{\phi}_{0} \frac{\mathrm{j}_{0}\left(\rho_{1}\right)}{3} \rho \cos \theta=\epsilon \hat{\phi}_{0} \frac{\mathrm{j}_{0}\left(\rho_{1}\right)}{3} \hat{z}(\rho, \theta) \equiv D_{0}(\rho, \theta), \\
\lim _{\rho \rightarrow \infty} \mathcal{D}_{1}(\rho, \theta, \varphi) & =0, \quad \lim _{\rho \rightarrow \infty} \mathcal{D}_{2}(\rho, \theta)=-\epsilon \hat{\phi}_{2} \frac{\mathrm{j}_{0}\left(\rho_{1}\right)}{3} \equiv D_{2}
\end{aligned}
$$

where the horizontal radius $r(\rho, \theta)=\rho \sin \theta$ and $\hat{z}(\rho, \theta)=\rho \cos \theta$. Thus, the far-field motion of mode- 0 is a rigid rotation with constant angular velocity $\Omega_{0}$, and the far-field motion of mode- 1 is a rigid translation with constant linear velocity $U_{1}$ given by

$$
\Omega_{0} \equiv \frac{\boldsymbol{U}_{0}(\rho, \theta) \cdot \boldsymbol{e}_{\varphi}}{\rho \sin \theta}=-\hat{\phi}_{0} \frac{\mathrm{j}_{0}\left(\rho_{1}\right)}{3}, \quad U_{1}=\hat{\phi}_{1} \frac{\mathrm{j}_{0}\left(\rho_{1}\right)}{3} .
$$

Mode-0 induces also a linear far-field density anomaly proportional to $\hat{z}$, while mode- 2 induces a constant density anomaly. Since the far-field flow of the steady state solution consists of a rigid horizontal motion and a constant vertical stratification we may obtain an unsteady isolated geopotential solution $\widetilde{\phi}(\rho, \theta, \varphi, t)$ having vanishing far-field flow and, at the same time, vanishing density anomaly, by adding a background geopotential $\bar{\phi}$, responsible for the background flow $\overline{\boldsymbol{r}}(\boldsymbol{X}, t)$ and background stratification, to the steady solution $\phi$, and compose this sum with the inverse background flow $\overline{\boldsymbol{r}}^{\iota}(\boldsymbol{x}, t)$ which provides the initial location (or location in the reference configuration) of a fictitious fluid particle $\boldsymbol{X}$ moved to $(\boldsymbol{x}, t)$ by the background flow $\overline{\boldsymbol{r}}(\boldsymbol{X}, t)$. Thus, the explicit expression for the unsteady geopotential $\widetilde{\phi}$ may be obtained as

$$
\widetilde{\phi}(\boldsymbol{x}, t)=(\phi+\bar{\phi})\left(\overline{\boldsymbol{r}}^{\iota}(\boldsymbol{x}, t)\right) .
$$

In the unsteady solution the dipole moves with angular velocity $\Omega \equiv-\Omega_{0}$ and linear velociy $U \equiv-U_{1}$, describing a circular trajectory with a signed radius of curvature $R=U / \Omega$, given by

$$
\Omega=\hat{\phi}_{0} \frac{\mathrm{j}_{0}\left(\rho_{1}\right)}{3}, \quad U=-\hat{\phi}_{1} \frac{\mathrm{j}_{0}\left(\rho_{1}\right)}{3}, \quad R=-\frac{\hat{\phi}_{1}}{\hat{\phi}_{0}} .
$$

These relations are identical to those for two-dimensional dipoles (Meleshko \& van Heijst 1994; Flierl et al. 1983; Viúdez 2019) except that here the modal amplitudes $\hat{\phi}_{n}$ are associated to the spherical Bessel functions $\mathrm{j}_{n}(\rho)$ rather than to the ordinary Bessel functions $\mathrm{J}_{n}(r)$.

In order to explicitly express the unsteady geopotential $\widetilde{\phi}(3.23)$ we introduce the background flow $\overline{\boldsymbol{r}}$ as a rigid motion with constant angular velocity $\Omega$ and linear velocity $\boldsymbol{U}$, as the solution to the differential equation

$$
\frac{\partial \overline{\boldsymbol{r}}}{\partial t}(\boldsymbol{X}, t)=\boldsymbol{U}+\Omega \boldsymbol{e}_{z} \times \overline{\boldsymbol{r}}(\boldsymbol{X}, t) .
$$

Assuming $\hat{\phi}_{0} \neq 0$ the solution to $(3.25)$ is

$$
\overline{\boldsymbol{r}}(\boldsymbol{X}, t)-\boldsymbol{e}_{z} \times \boldsymbol{U} / \Omega=\boldsymbol{R}[\Omega t] \cdot\left(\boldsymbol{X}-\boldsymbol{e}_{z} \times \boldsymbol{U} / \Omega\right),
$$

where $\boldsymbol{R}[\theta]$ is the $2 \mathrm{D}$ rotation matrix. Transformation (3.26) is a rotation, by an angle $\Omega t$, of vector $\boldsymbol{X}-\boldsymbol{e}_{z} \times \boldsymbol{U} / \Omega$, that is, point $\boldsymbol{X}$ rotates along a circle centered at point $\boldsymbol{e}_{z} \times \boldsymbol{U} / \Omega$. The inverse function $\overline{\boldsymbol{r}}^{\iota}$, obtained from (3.26), is

$$
\overline{\boldsymbol{r}}^{\iota}(\boldsymbol{x}, t)=\boldsymbol{R}^{\top}[\Omega t] \cdot\left(\boldsymbol{x}-\boldsymbol{e}_{z} \times \boldsymbol{U} / \Omega\right)+\boldsymbol{e}_{z} \times \boldsymbol{U} / \Omega .
$$

The unsteady geopotential $\widetilde{\phi}(\rho, \theta, \varphi, t)$ is obtained by adding to the steady geopotential $\phi(\rho, \theta, \varphi)$ the background geopotential

$$
\bar{\phi}(\rho, \theta, \varphi) \equiv \bar{\phi}_{0}(\rho)+\bar{\phi}_{1}(\rho, \theta, \varphi)+\bar{\phi}_{2}(\rho, \theta, \varphi)
$$


where the modal background geopotentials are

$$
\begin{aligned}
\frac{\bar{\phi}_{0}(\rho)}{\hat{\phi}_{0}} & \equiv \frac{\mathrm{j}_{0}\left(\rho_{1}\right)}{6} \rho^{2}, \\
\frac{\bar{\phi}_{1}(\rho, \theta, \varphi)}{\hat{\phi}_{1}} & \equiv-\frac{\mathrm{j}_{0}\left(\rho_{1}\right)}{3} \rho \sin \theta \cos \varphi=-\frac{\mathrm{j}_{0}\left(\rho_{1}\right)}{3} x(\rho, \theta, \varphi), \\
\frac{\bar{\phi}_{2}(\rho, \theta, \varphi)}{\hat{\phi}_{2}} & \equiv-\frac{\mathrm{j}_{0}\left(\rho_{1}\right)}{3} \rho \cos \theta=-\frac{\mathrm{j}_{0}\left(\rho_{1}\right)}{3} \hat{z}(\rho, \theta) .
\end{aligned}
$$

The background geopotential $\bar{\phi}_{0}(\rho)$ adds to the steady flow the required vertical vorticity $\bar{\zeta}_{0}^{g}=2 \Omega=(2 / 3) \hat{\phi}_{0} \mathrm{j}_{0}\left(\rho_{1}\right)$ (see $\left.(3.12)\right)$ and background stratification $\overline{\mathcal{S}}_{0}=(1 / 3) \hat{\phi}_{0} \mathrm{j}_{0}\left(\rho_{1}\right)$ (see $(3.13)$ ), adding up a constant background PVA $\bar{\varpi}_{0}^{q}=\hat{\phi}_{0} \mathrm{j}_{0}\left(\rho_{1}\right)$ to the steady flow (3.7), such that the exterior PVA of the unsteady flow vanishes. The background geopotential $\bar{\phi}_{1}$ adds the required velocity $\overline{\boldsymbol{u}}_{1}^{g}=-(1 / 3) \hat{\phi}_{1} \mathrm{j}_{0}\left(\rho_{1}\right) \boldsymbol{e}_{y}$ to cancel the horizontal velocity at infinity (3.9). Finally the background geopotential $\bar{\phi}_{2}$ adds the background vertical displacement of isopycnals $\overline{\mathcal{D}}_{2}=(1 / 3) \hat{\phi}_{1} \epsilon \mathrm{j}_{0}\left(\rho_{1}\right)$ such that this modal solution has finite mass anomaly. Figures 1,2 , and 3 show the unsteady distributions at $t_{0}=0$. The pattern of the QG vertical velocity $w^{q}$ (figure 3) remains unchanged since the $w^{q}$ distribution is only rigidly advected by the background flow. Though in the unsteady state (3.14) is not longer valid when applied to the unsteady velocity $\widetilde{\boldsymbol{u}}^{g}(\boldsymbol{x}, t)$ and unsteady density anomaly $\widetilde{\mathcal{D}}(\boldsymbol{x}, t)$, the QG vertical velocity $w^{q}$ always obeys the QG omega equation $\hat{\nabla}^{2} \widetilde{w}^{q}=2 \nabla_{h} \cdot\left(\nabla_{h} \widetilde{\boldsymbol{u}}^{g} \cdot \boldsymbol{\nabla}_{h} \widetilde{\mathcal{D}}\right)$, whose right-hand side is invariant to the rigid velocity and constant stratification of the background flow.

The fundamental quantity, however, is the QG PVA $\widetilde{\varpi}^{q}(\boldsymbol{x}, t)$ which at $t_{0}=0$ is

$$
\widetilde{\varpi}^{q}\left(\rho, \theta, \varphi, t_{0}\right)=-\hat{\phi}_{0}\left(\mathrm{j}_{0}(\rho)-\mathrm{j}_{0}\left(\rho_{1}\right)\right)-\hat{\phi}_{1} \mathrm{j}_{1}(\rho) \sin \theta \cos \varphi-\hat{\phi}_{2} \mathrm{j}_{1}(\rho) \cos \theta,
$$

for $\rho \leqslant \rho_{1}$, and zero elsewhere.

\section{Concluding remarks}

We have provided a QG solution of a baroclinic dipole. This 3D vortex model involves three PVA modes, each one having its own independent amplitude. These three amplitudes $\left\{\hat{\phi}_{1}, \hat{\phi}_{2}, \hat{\phi}_{3}\right\}$, together with the ratio $c \equiv N / f$, determine the parameter space of the vortex. The dipolar mode provides the constant speed of displacement of the vortex and is necessary for the existence of vertical velocity. The spherical mode provides the horizontal asymmetry and is responsible for the constant curvature of the dipole's trajectory. The vertical tilting mode provides vortex vertical asymmetry and its interaction with the dipolar mode develops an additional vertical velocity field.

For the present results to remain physically acceptable within the QG approximation it is required that modal amplitudes $\left|\hat{\phi}_{n}\right| \ll 1$. Application to more ageostrophic fields, beyond the QG approximation, seems to be quite complicated, in part because largely ageostrophic dipoles spontaneously generate inertia-gravity waves which break the rigidity condition of the unsteady PVA contour solutions obtained here.

Though additional azimuthal modes $m \geqslant 2$ are dispersive in the sense that involve dipoles moving along different directions, modes of order $m=0$ and degree $l>2$ might be stable. Investigation along this line of research is left for future work.

I thank three anonymous referees for their very helpful comments. Partial support for 
this study was obtained through project CTM2014-56987-P (Spanish Ministry of Science and Innovation).

\section{Appendix A. Mass conservation}

In stable stratified fluids it is convenient to express the density anomaly as $\rho^{\prime}(\boldsymbol{x}, t) \equiv$ $\rho(\boldsymbol{x}, t)-\varrho_{Z} z-\rho_{0}$, where $\rho(\boldsymbol{x}, t)$ is the mass density, with $\rho_{0}$ and $\varrho_{Z}$ are a constant background density and a constant background density stratification that do not need to be specified. Furthermore, it is also convenient to express the density $\rho(\boldsymbol{x}, t)$ in terms of the field $d(\boldsymbol{x}, t) \equiv\left(\rho(\boldsymbol{x}, t)-\rho_{0}\right) / \varrho_{Z}$, which represents the depth, or vertical location, that an isopycnal located at $\boldsymbol{x}$ at time $t$ has in the reference configuration defined by $\varrho_{Z} z+\rho_{0}$. Thus, the density field is expressed in terms of distances. The displacement $\mathcal{D}(\boldsymbol{x}, t)$ of isopycnals with respect to the reference density configuration is

$$
\mathcal{D}(\boldsymbol{x}, t) \equiv z-d(\boldsymbol{x}, t) .
$$

$\mathcal{D}(\boldsymbol{x}, t)$ is the vertical displacement of the isopycnal currently located at $(\boldsymbol{x}, t)$ with respect to its position in the reference configuration. Density conservation in QG dynamics is

$$
\frac{\partial \mathcal{D}}{\partial t}+\boldsymbol{u}^{g} \cdot \nabla_{h} \mathcal{D}-w^{q}=0
$$

where $w^{q}$ is the QG vertical velocity. The vertical density stratification anomaly is defined as $\mathcal{S}(\boldsymbol{x}, t) \equiv-\partial \mathcal{D}(\boldsymbol{x}, t) / \partial z=\partial d(\boldsymbol{x}, t) / \partial z-1=\varrho_{Z}^{-1} \partial \rho(\boldsymbol{x}, t) / \partial z-1$, so that $\mathcal{S}>-1$ is required for stable stratification and $\mathcal{S}=-1$ implies neutral stratification $(\partial \rho / \partial z=0)$.

\section{Appendix B. Azimuthal modes and Helmholtz equation}

Assuming azimuthal modes in spherical coordinates $\phi(\rho, \theta, \varphi)=G(\rho, \theta) \mathrm{e}^{\mathrm{i} m \theta}$, conservation of QG PVA (2.1) in the steady state $\boldsymbol{u}^{g} \cdot \boldsymbol{\nabla} \varpi^{q}=0$ (hat symbol ${ }^{\wedge}$ omitted here for simplicity) leads to

$$
\left(\boldsymbol{e}_{z} \times \frac{\nabla G}{G}-\frac{\mathrm{i} m}{\rho \sin \theta} \boldsymbol{e}_{r}\right) \cdot\left(\frac{\nabla H}{H}+\frac{\mathrm{i} m}{\rho \sin \theta} \boldsymbol{e}_{\varphi}\right)=0,
$$

where $H(\rho, \theta) \equiv \nabla^{2} G-m^{2} F(\rho, \theta) /\left(\rho^{2} \sin ^{2} \theta\right)$, and the horizontal radial unit vector $\boldsymbol{e}_{r}=\sin \theta \boldsymbol{e}_{\rho}+\cos \theta \boldsymbol{e}_{\theta}$. Using $\boldsymbol{e}_{z} \times \boldsymbol{\nabla} G=G_{r} \boldsymbol{e}_{\varphi}$, where, as usual, $G_{r}=$ $\partial_{r} G(\rho(r, \varphi, z), \theta(r, \varphi, z))=\boldsymbol{e}_{r} \cdot \boldsymbol{\nabla} G$ is the horizontal radial derivative, we obtain

$$
\frac{G_{r}}{G}=\frac{H_{r}}{H} \quad \Rightarrow \quad-k^{2} G=\nabla^{2} G-\frac{m^{2}}{\rho^{2} \sin ^{2} \theta} G,
$$

where $k$ is an integration constant. Multiplication of (B 2) by $\mathrm{e}^{\mathrm{i} m \varphi}$ leads to the Helmholtz equation $\nabla^{2} \phi=-k^{2} \phi(3.1)$.

\section{Appendix C. Spherical Bessel functions}

The spherical Bessel functions of degrees $l=0, l=1, l=2$, and $l=3$ used here are

$$
\begin{aligned}
& \mathrm{j}_{0}(\rho)=\frac{\sin \rho}{\rho}, \quad \mathrm{j}_{1}(\rho)=\frac{\sin \rho}{\rho^{2}}-\frac{\cos \rho}{\rho}, \\
& \mathrm{j}_{2}(\rho)=\frac{\left(3-\rho^{2}\right) \sin \rho}{\rho^{3}}-\frac{3 \cos \rho}{\rho^{2}}, \quad \mathrm{j}_{3}(\rho)=\frac{\left(\rho^{2}-15\right) \cos \rho}{\rho^{3}}-\frac{3\left(2 \rho^{2}-5\right) \sin \rho}{\rho^{4}} .
\end{aligned}
$$


The following equalities $\mathrm{j}_{2}\left(\rho_{1}\right)=-\mathrm{j}_{0}\left(\rho_{1}\right), \mathrm{j}_{3}\left(\rho_{1}\right)=-5 \mathrm{j}_{0}\left(\rho_{1}\right) / \rho_{1}$ have been also used, as well as the differential relation

$$
\left(\frac{1}{\rho} \frac{d}{d \rho}\right)^{m}\left(\rho^{-n} j_{n}(\rho)\right)=(-1)^{m} \rho^{-n-m} j_{n+m}(\rho),
$$

for $n=0, \pm 1, \pm 2, \ldots$.

\section{REFERENCES}

Ahlnäs, K., Royer, T. C. \& George, T. H. 1987 Multiple dipole eddies in the Alaska Coastal Current detected with Landsat thematic mapper data. J. Geophys. Res. Oceans 92 (C12), 13041-13047.

Cavallini, F. \& Crisciani, F. 2013 Quasi-Geostrophic Theory of Oceans and Atmosphere. Springer Netherlands.

Chaplygin, S. A. 1903 One case of vortex motion in fluid. Trans. Phys. Sect. Imperial Moscow Soc. Friends of Natural Sciences 11 (N 2), 11-14.

Cunningham, P. \& Keyser, D. 2000 Analytical and numerical modelling of jet streaks: Barotropic dynamics. Quarterly Journal of the Royal Meteorological Society 126 (570), 3187-3217.

Fedorov, K. N. \& Ginsburg, A. I. 1989 Mushroom-like currents (vortex dipoles): one of the most widespread forms of non-stationary coherent motions in the ocean. In Mesoscale/Synoptic Coherent Structures in Geophysical Turbulence (ed. J. C. J. Nihoul \& B. M. Jamart), Elsevier Oceanography Series, vol. 50, pp. 1-14. Elsevier.

Flierl, G. R., Larichev, V. D., McWilliams, J. C. \& Reznik, G. M. 1980 The dynamics of baroclinic and barotropic solitary eddies. Dynamics of Atmospheres and Oceans $\mathbf{5}$ (1), $1-41$.

Flierl, G. R., Stern, M. E. \& Whitehead, J. A. 1983 The physical significance of modons: Laboratory experiments and general integral constraints. Dyn. Atmos. Oceans 7, 233-263.

Kloosterziel, R.C., Carnevale, G.F. \& Phillippe, D. 1993 Propagation of barotropic dipoles over topography in a rotating tank. Dyn. Atmos. Oceans 19, $65-100$.

Meleshro, V. V. \& van Heijst, G. J. F. 1994 On Chaplygin's investigations of twodimensional vortex structures in an inviscid fluid. J. Fluid Mech. 272, 157-182.

de Ruijter, W. P. M., van Aken, H. M., Beier, E. J., Lutjeharms, J. R. E., Matano, R. P. \& Schouten, M. W. 2004 Eddies and dipoles around South Madagascar: formation, pathways and large-scale impact. Deep Sea Res. I 51, 383-400.

Velasco Fuentes, O. U. \& van Heijst, G. J. F. 1995 Collision of dipolar vortices on a $\beta$ plane. Phys. Fluids 7, 2735-2750.

Viúdez, A. 2019 Azimuthal-mode solutions of two-dimensional Euler flows and the ChaplyginLamb dipole. J. Fluid Mech. 859, R1.

Voropayev, S. I. \& Afanasyev, Ya. D. 1992 Two-dimensional vortex-dipole interactions in a stratified fluid. J. Fluid Mech. 236, 66-689. 